Document downloaded from:

http://hdl.handle.net/10251/57073

This paper must be cited as:

Rius Mercado, M.; Bolea Boluda, M.; Mora Almerich, J.; Ortega Tamarit, B.; Capmany Francoy, J. (2014). Multiband-UWB Signals Generation Based on Incoherent Microwave Photonic Filters. IEEE Photonics Technology Letters. 26(2):142-145. doi:10.1109/LPT.2013.2290315.

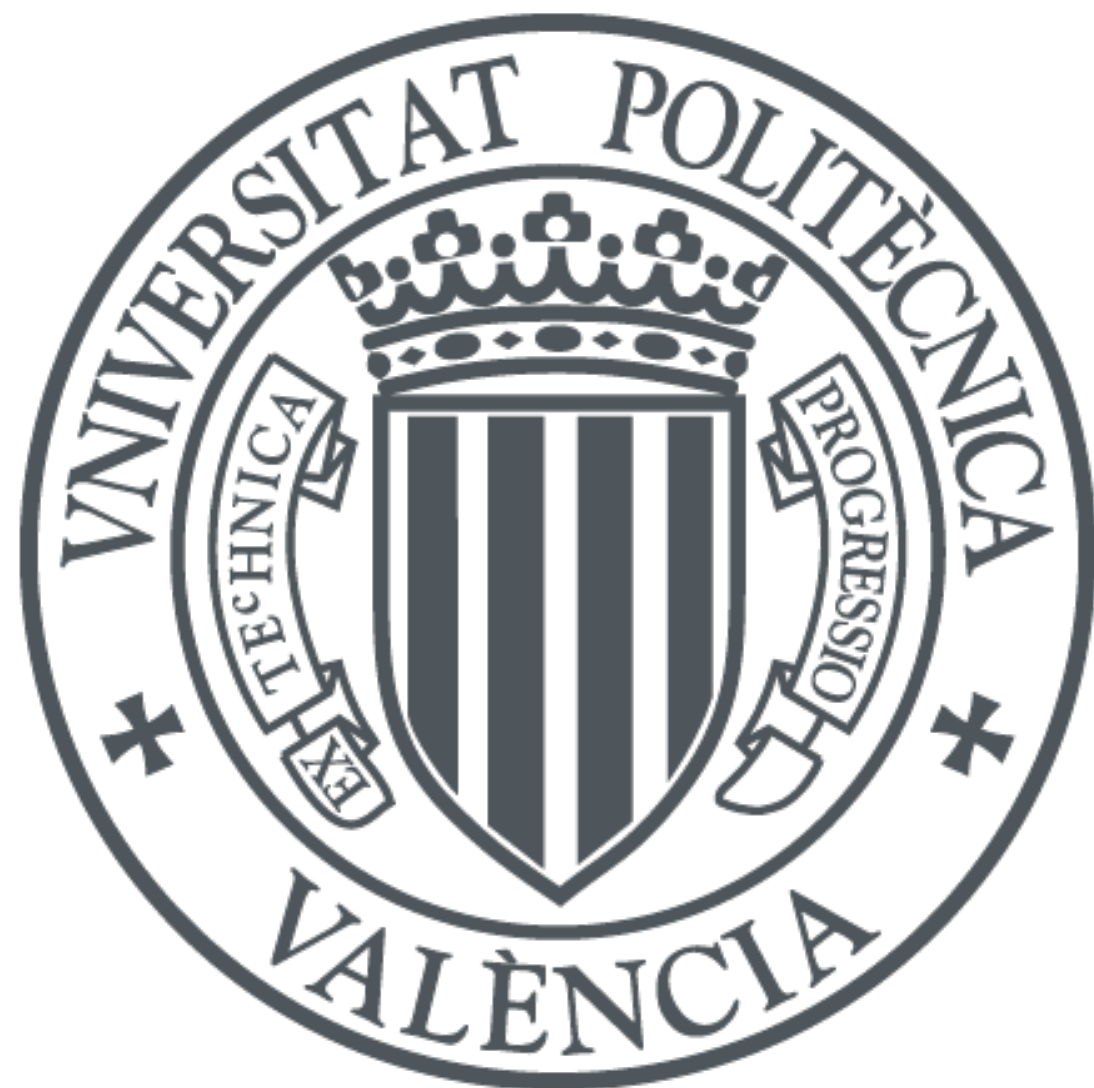

The final publication is available at

http://dx.doi.org/10.1109/LPT.2013.2290315

Copyright Institute of Electrical and Electronics Engineers (IEEE)

Additional Information

"C (C) 20xx IEEE. Personal use of this material is permitted. Permission from IEEE must be obtained for all other uses, in any current or future media, including reprinting/republishing this material for advertising or promotional purposes, creating new collective works, for resale or redistribution to servers or lists, or reuse of any copyrighted component of this work in other works." 


\title{
Multiband-UWB signals generation based on incoherent microwave photonic filters
}

\author{
Manuel Rius, Mario Bolea, Jose Mora, Beatriz Ortega, Member, IEEE and José Capmany, Fellow, \\ IEEE.
}

\begin{abstract}
A structure for Multiband-UWB waveforms generation is proposed using a $\mathbf{N}$ tap microwave photonic filter with positive and negative taps. The system is based on phase inversion in electro-optical modulators and the sliced broadband source optical signal by means of a structure of AWGs which permits to obtain a high number of taps. System performance is experimentally shown by the generation of an uniform waveform. Capabilities as reconfigurability and frequency tuning have been also experimentally demonstrated. The flexibility of the system allows to obtain Multiband UWB waveforms which fulfill the FCC spectral requirements.
\end{abstract}

Index Terms-Microwave photonic filter, Multiband-UWB technology, Signal processing, Reconfigurability, Frequency tuning.

\section{INTRODUCTION}

$\mathrm{U}$ LTRAWIDEBAND (UWB) technologies for short-range high-speed wireless communications and sensor networks have attracted considerable interest in recent years owing to some unique advantages, such as low power consumption, high data rate and immunity to multipath fading [1, 2]. The U.S. Federal Communication Commission (FCC) has regulated the spectral band from 3.1 to $10.6 \mathrm{GHz}$ for the unlicensed use of UWB with a transmitted power spectral density of less than $41.3 \mathrm{dBm} / \mathrm{MHz}$ for indoor wireless communications. In practical terms, this means that UWB signals must fulfill a spectral mask. Two different UWB signaling formats can be distinguished, Impulse-Radio UWB (IR-UWB) and Multiband UWB (MB-UWB), depending on the spectral distribution of the signals within the FCC mask. For IR-UWB, the signals correspond to shorter pulses whose electrical bandwidth is extended within the whole FCC mask. However, MB-UWB signaling format divides the available electrical spectrum in different narrower channels leading to longer pulses in time domain [3]. In both cases, the limitation in terms of density power reduces the coverage distance of UWB signals. To overcome the UWB short-range wireless transmission limitation, UWB-over-fiber technology has emerged to increase the propagation distance of wireless signal. Therefore,

Manuscript received September 3, 2013. The research leading to these results has received funding from the national project TEC2011-26642 (NEWTON) funded by the Ministerio de Ciencia y Tecnología and the regional project PROMETEO GVA 2013/12 Next Generation Microwave Photonic technologies.

M. Rius, M. Bolea, J. Mora, B. Ortega and J. Capmany are with the ITEAM Research Institute, Universidad Politécnica de Valencia, Valencia, Spain (e-mail: jmalmer@iteam.upv.es). photonic operation of UWB signals is attractive since it can be directly transmitted along the fiber link [4]. Moreover, photonic UWB generation involves the inherent advantages of operating in the optical domain such as: low loss, small size or immunity to electromagnetic interferences (EMI) [5].

In literature, a great number of approaches related to UWB generation in the optical domain have been proposed. We can find schemes to generate UWB pulses based on phasemodulation-to-intensity-modulation (PM-IM) using an optical frequency discriminator [6] or a fiber link combined with fourwave mixing [7]. Optical spectral shaping and frequency-totime mapping has been also proposed for UWB signals generation using a single mode fiber link for the conversion [8, 9]. One of the most popular techniques proposed for UWB pulses generation recently corresponds to microwave photonic filtering. In this sense, we found structures based on cross-gain modulation in Semiconductor Optical Amplifiers (SOA) [9], the nonlinear amplification process in a SOA [10], a phase modulator and an asymmetric Mach-Zehnder Interferometer (AMZI) [11] and, also, based on phase inversion in electrooptic modulator combined with a dispersive element [12].

The most of photonic proposals that can be found in the literature are focused in the generation of IR-UWB pulses. As far as we know, a reduced number of structures have been proposed to MB-UWB based on hybrid photonic microwave filter [13], synchronously-apodized polarization modulation and birefringence time delay [14] or single bandpass microwave photonic filter with broadband source, a MachZehnder interferometer and dispersive elements [15].

In this paper, we propose a MB-UWB generator using a microwave photonic filter based on the processing of incoherent optical samples by a dispersive element and featuring positive and negative sample polarity by using phase inversion in electro-optical modulators. This scheme has been previously presented in [16] for IR-UWB. Nevertheless, the possibility of generating MB-UWB signals has been experimentally demonstrated since a high number of taps can be achieved by the slicing of a broadband source. Different structure capabilities have been also proved. The reconfigurability of the envelope waveform is achieved by the control of each optical sample independently which permits to fulfill the FCC spectral mask. Moreover, the frequency tuning can be carried out by changing the dispersion induced by the fiber link in the system. Therefore, this system allows to generate signals compatible with MB-UWB technology. 
Comparing with previous configurations [15], the stability of the waveforms generated has been improved since noninterferometric structures are used.

\section{GENERATOR SETUP AND OPERATION PRINCIPLE}

Fig. 1 shows the proposed experimental setup to generate Multiband UWB pulses. This generator is based on a N-tap microwave photonic filter with positive and negative coefficients. The system operation principle can be found in [12]. The novelty of this scheme compared with [12] is the use of incoherent broadband laser source instead of multiple lasers. This fact increases considerably the capacity and flexibility of UWB pulses generator.

In this case, the filter taps have been obtained by slicing the output signal of an Amplified Spontaneous Emission (ASE) source using a structure composed by three Array Waveguide Gratings (AWGs) set in two stages. Firstly, the incoherent optical source output signal is demultiplexed by an AWG in the first stage. Each channel is launched to one of the AWGs (AWG1 or AWG2) in the second stage, depending on the cross or bar state of $2 \times 2$ optical switches. As a result, a group of channels are multiplexed in a fiber ready to be launched into one of the electro-optic modulators (EOM). Note that each optical output of the AWG in the first stage contains a variable attenuator which permits to control the power of each wavelength channel independently. In our case, for the experimental results, we have used $1 \times 40$ channel AWGs with equal spectral response placed symmetrically with $0.4 \mathrm{~nm}$ bandwidth and a standard ITU spacing channels of 0.8 $\mathrm{nm}$. In this way, the optical spectrum of the concatenated channel has a $0.31 \mathrm{~nm}$ bandwidth for a given channel.

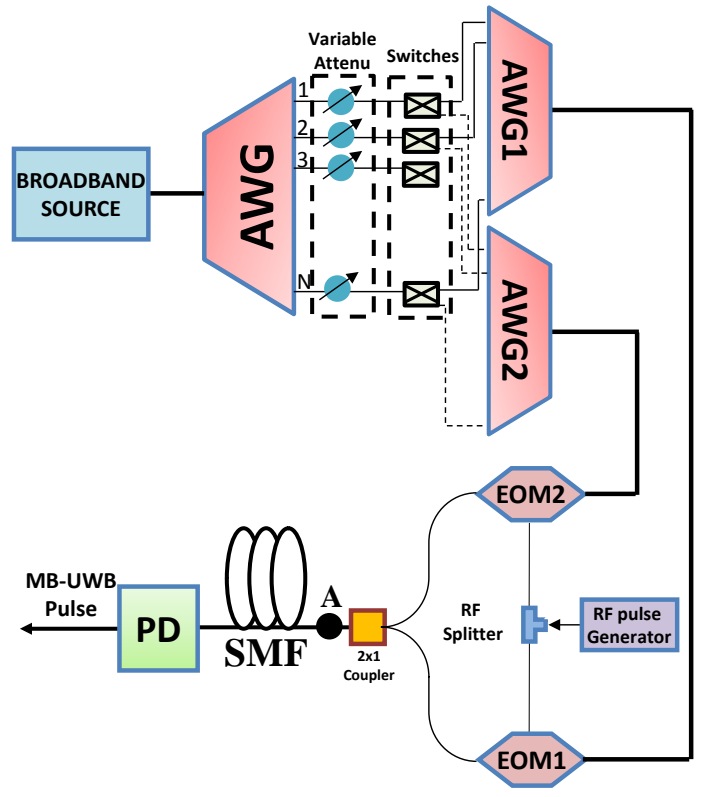

Fig. 1. Layout of the Multiband-UWB generator.

The optical channels at the output of AWG1 and AWG2 are launched into two EOMs (EOM1 and EOM2), biased in opposite slopes region and both modulated by the same electrical pulse using a RF splitter. In this way, the optical pulses at EOM1 and EOM2 output port show positive and negative polarities, respectively. The electrical input signal is generated by an electrical pulse generator with a pattern of one "1" and sixty-three "0" (total 64 bits) and $12.5 \mathrm{~Gb} / \mathrm{s}$ bit rate.

Then, the modulated optical signals are coupled and launched into a single mode fiber (SMF) link which introduces a different delay between each sample according to the central wavelength of the corresponding channel. The delay undergone by the optical pulses $\left(\tau_{n}\right)$ is approximated by [12]:

$$
\tau_{\mathrm{n}} \approx \beta_{2} \mathrm{~L}\left(\omega_{\mathrm{n}}-\omega_{0}\right)
$$

where $\beta_{2}$ and $L$ corresponds to the first order dispersion parameter and length of the SMF, respectively; $\omega_{\mathrm{n}}$ corresponds to the central optical frequency of the n-channel. According to Eq. 1, the time delay difference between two adjacent optical pulses $\left(\Delta \tau=\tau_{n}-\tau_{n-1}\right)$ is given by the optical frequency spacing between the optical channels and the fiber dispersion. The Free Spectral Range (FSR) of the equivalent microwave photonic filter responds to:

$$
\operatorname{FSR}=1 / \Delta \tau
$$

Therefore, the central frequency of the waveform generated is related to the optical frequency spacing between the selected channels and the dispersion induced in the system. A standard SMF-28 is used in the experimental setup with a $\beta_{2}=-22$ $\mathrm{ps}^{2} / \mathrm{km}$ and a length of $5 \mathrm{Km}$. In case of higher fiber dispersion, compensating dispersion modules would be needed to manage it in order to obtain the required $\Delta \tau$. Finally, optical output signal is detected by a photodetector (PD).

Fig. 2 plots the optical modulated pulses at the output of both EOMs and after propagation over the fiber link. The system has been analyzed by selecting the EOM1 or EOM2 when only a channel centered at $1546.92 \mathrm{~nm}$ is selected. We can observe that pulse broaded by fiber propagation due to chromatic dispersion is shown to be negligible [12]. Note as a positive and negative polarity pulse is obtained depending on the bias point of the modulator. As can be observed, the full width half maximum (FWHM) of pulses is practically the same before and after the transmission through the fiber. Therefore, the chromatic dispersion effects over the optical channel are neglected, while a delay is introduced between the channels.
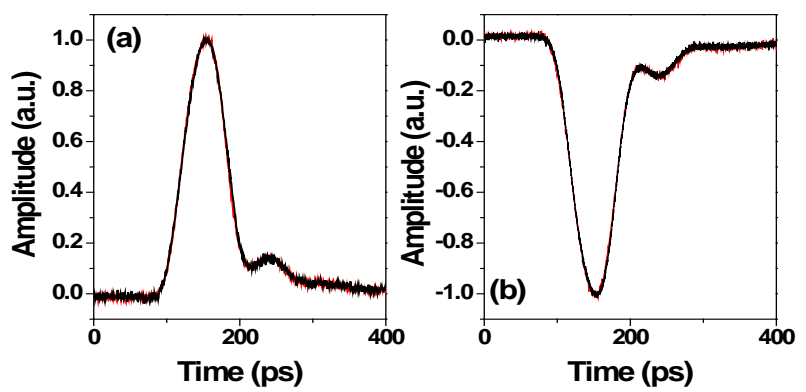

Fig. 2. Optical pulses at the input (dashed line) and output (solid line) of the fiber link coming from (a) EOM 1 and (b) EOM 2.

\section{EXPERIMENTAL RESULTS}

\section{A. System performance.}

Firstly, the performance of the Multiband UWB generation system is experimentally demonstrated. In this case, 11 equalized channels with the optical source power distribution 
shown in Fig. 3a, with a 3-dB bandwidth of $8 \mathrm{~nm}$ (measured at point $\mathrm{A}$ of the scheme). These channels have been alternatively introduced in each EOM. The resulting waveform is plotted in Fig. 3b which presents an uniform envelope according to the optical source power distribution. Since each optical channel has been alternatively introduced in the modulators, the signal generated has both positive and negative polarities. The electrical power spectrum corresponding to the generated waveform is presented in Fig. 3c. From the experimental parameters specified in the previous section, we experimentally check that the central frequency of the signal generated is around $6.85 \mathrm{GHz}$ which corresponds to the center of the FCC mask. Moreover, as can be observed, the spectral content around baseband component is reduced since positive and negative taps have been introduced in the system [12]. Nevertheless, the signal generated does not fulfill the FCC spectral requirements due to the spectral component around GPS band. Therefore, the signal power should be reduced which implies an inefficient use of the UWB spectrum.
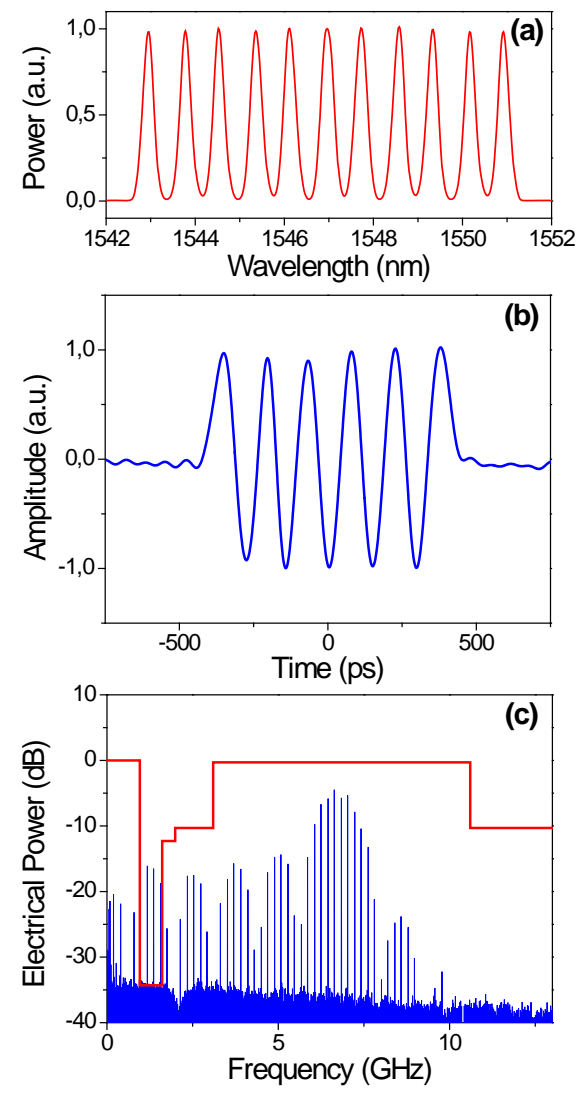

Fig. 3. Experimental results of the (a) optical source power spectral density, (b) generated waveform and (c) corresponding electrical spectrum -FCC mask is also shown-.

\section{B. Generator Capabilities}

In the following, we are going to show the capacity of the scheme for controlling the envelope and tuning the central frequency of the generated signal. Firstly, the waveform reconfigurability is experimentally proved by the results of Fig. 4. In this case, the optical source is launched into the first AWG to provide a set of optical carriers. The Gaussian profile is performed by means of a variable optical attenuator (VAO) module which controls the attenuation of each channel with a $35 \mathrm{~dB}$ tuning range. A total of 23 channels are selected and alternatively introduced in each EOM. The power of each channel is adjusted by the attenuators according to a Gaussian profile with a 3-dB optical bandwidth $(\delta \lambda)$ of $8 \mathrm{~nm}$.
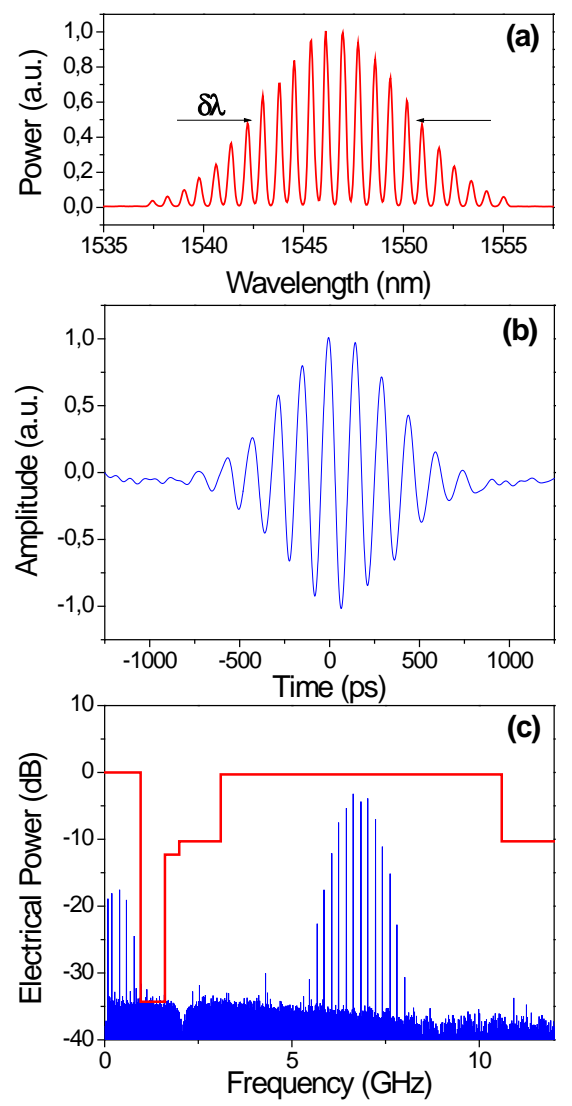

Fig. 4. Experimental results of the (a) optical source power spectral density, (b) waveform and (c) corresponding electrical spectrum for a Gaussian profile with a 3-dB bandwidth of $8 \mathrm{~nm}$-FCC mask is also shown-.

The optical signal power distribution measured at the point A in the scheme is show in Fig. 4a. The resulting waveform and its corresponding electrical power spectrum are shown in Figs. 4b and 4c, respectively. As can be observed, the envelope of the waveform generated presents a Gaussian behaviour according to the optical source power distribution. The corresponding spectrum of the generated signal fulfils the FCC mask since the spectral content around the GSP band has been removed. Therefore, we have experimentally demonstrated the viability of our system for generating Multiband UWB waveforms according to the FCC spectral requirements. This fact permits to fit the waveforms to the FCC mask avoiding the need of reducing the signal power and achieving an efficient solution in terms of power spectral density with the only restriction of the system noise.

Finally, tuning frequency capability has been also demonstrated. As we have pointed out in section II, the central frequency of the generated waveform is related to the frequency spacing of the optical channels and the dispersion induced by the system according to Eqs. 1 and 2. The optical source power distribution is maintained with 23 channels and a 
3-dB optical bandwidth $(\delta \lambda)$ of $8 \mathrm{~nm}$, as shows Fig. $5 \mathrm{~b}$. In this case, $8 \mathrm{~km}$ standard SMF-28 link is used. The generated waveform and its corresponding electrical power spectrum are shown in Figs. 5b and 5c, respectively. As we can observe, the generated signal envelope characteristics, both in terms of waveform and spectrum, are maintained respect to Fig. 4. Nevertheless, the signal frequency has been changed to 4.75 $\mathrm{GHz}$, according to Eqs. 1 and 2. Therefore, we have experimentally demonstrated the capability for tuning the frequency of the generated waveform by changing the dispersion induced by the system. In the literature, we can find proposals where a discrete control of the dispersion in the system is achieved using different SMF links and MEMS [17]. Note that all measurements show the electrical power normalized to an output power of $-41.3 \mathrm{dBm} / \mathrm{MHz}$ according to the requirements of the FCC mask.
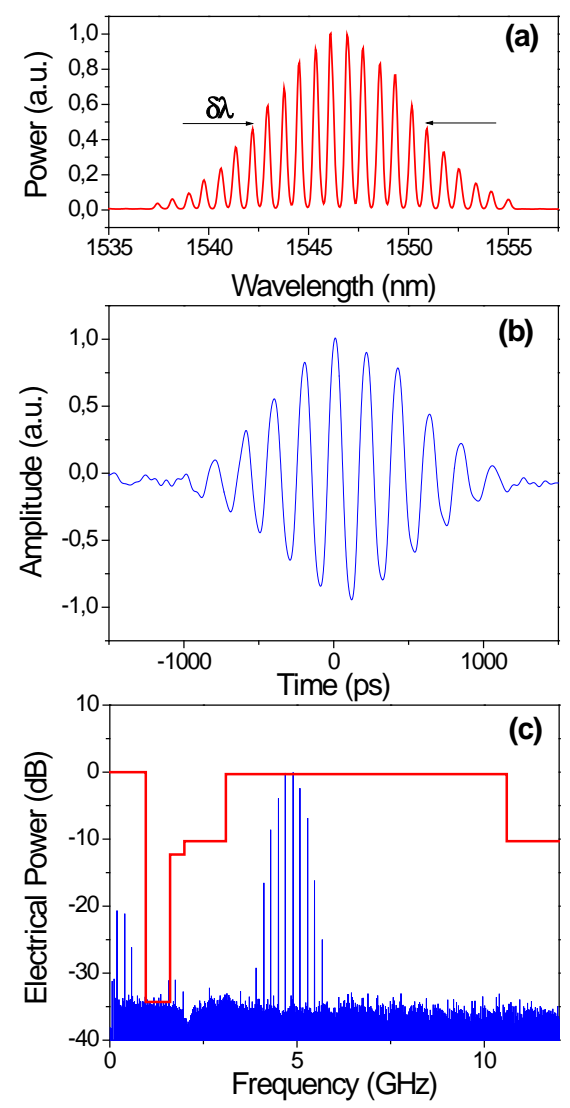

Fig. 5. Experimental results of the (a) optical source power spectral density, (b) waveform and (c) corresponding electrical spectrum for a Gaussian profile with a 3-dB bandwidth of $8 \mathrm{~nm}$ and a SMF link of $8 \mathrm{~km}$-FCC mask is also shown.

\section{CONCLUSIONS}

In conclusion, we have experimentally demonstrated an UWB waveform generator using a $\mathrm{N}$ tap microwave photonic filter based on the slicing of an incoherent optical source and featuring positive and negative taps by means of polarity inversion in electro-optical modulators. The use of a broadband source is the key to increase considerably the capacity of the UWB generator while keeping reasonable costs. Indeed, multiband UWB signals have been experimentally demonstrated with a large number of optical taps due to the use of an optical broadband source. Different capabilities of the system have been also demonstrated as the envelope reconfigurability, central frequency tuning and the suppression of the baseband component. These capabilities enable to propose the generated multiband waveforms perfectly fulfilling the FCC spectral requirements.

\section{REFERENCES}

[1] G. R. Aiello, and G. D. Rogerson, "Ultra-wideband wireless systems," IEEE Microwave Mag., vol. 4, pp. 36-47, June 2003.

[2] D. Porcino, and W. Hirt, "Ultra-wideband radio technology: potential and challenges ahead,” IEEE Comm. Mag., vol 41, pp. 66-74, July 2003.

[3] L. Yanga, and G. B. Giannakis, "Ultra-Wideband communications: an idea whose time has come,” IEEE Signal Proces. Mag., vol 21, pp. 2654, November 2004

[4] J. Yao, "Photonics for ultrawideband communications," IEEE Microw. Mag., vol. 10, pp. 82-95, June 2009.

[5] J. Capmany, and D. Novak, "Microwave photonics combines two worlds," Nature Photonics, vol. 1, pp 319-329, June 2007.

[6] J. Shao, and J. Sun, "Photonic ultrawideband impulse radio shape modulation based on dual fiber tuning,” Opt. Lett., vol. 38, pp. 232-234, Jan. 2013.

[7] W. Li, L. X. Wang, W. Hofmann, N. H. Zhu, and D. Bimberg, "Generation of ultra-wideband triplet pulses on four-wave mixing and phase-to-intensity modulation conversion,” Opt. Exp., vol. 20, pp. 20222-20227, July 2012.

[8] M. Abtahi, and L. A. Rusch, "Arbitrary waveform generator supporting OOK, PPM and PSK modulation formats," in Proc. of IEEE Conference on Microwave Photonics (MWP'10, Montreal), pp. 294297.

[9] C. Wang, F. Zeng, and J. Yao, “All-fiber ultrawideband pulse generation based on spectral shaping and dispersion-induced frequency-to-time conversion," IEEE Photon. Tech. Lett., vol. 19, pp. 137-139, February 2006.

[10] Q. Wang, F. Zeng, S. Blais, and J. Jao, "Optical ultrawideband monocycle pulse generation based on cross-gain modulation in a semiconductor optical amplifier,” Opt. Lett., vol. 31, pp. 3083-3085, November 2006.

[11] Y. Yu, J. Dong, Z. Li, and X. Zhang, "UWB monocycle generation and bi-phase modulation based on Mach-Zehnder modulator and semiconductor optical amplifier," IEEE Photon. J., vol. 4, pp. 327-339, April 2012.

[12] M. Bolea, J. Mora, B. Ortega, and J. Capmany, “Optical UWB pulse generator using an $\mathrm{N}$ tap microwave photonic filter and phase inversion adaptable to different pulse modulation formats," Opt. Exp., vol. 17, pp. 5023-5032, March 2009.

[13] H. Chen, T. Wang, M. Li, M. Chen, and S. Xie, "Optically tunable multiband UWB pulse generation,” Opt. Exp., vol. 8, pp. 7447-7451, May 2008.

[14] H. Chen, C. Qiu, M. Chen, and S. Xie, "Multiband UWB pulse generation using hybrid photonic microwave filters," In Optical Fiber Communication Conference (OFC’08, San Diego), paper JThA66.

[15] M. Bolea, J. Mora, B. Ortega, and J. Capmany, "Photonic arbitrary waveform generation applicable to Multiband UWB communications," Opt. Exp., vol. 18 , pp. 26259-26267, December 2010.

[16] M. Bolea, J. Mora, B. Ortega, and J. Capmany, "High-order UWB pulse generation based on a microwave photonic filter using incoherent optical sources," in Proc. of IEEE Conference on Microwave Photonics (MWP'11, Singapore), pp. 462-465.

[17] J. Capmany, J. Mora, B. Ortega and D. Pastor, "Microwave photonic filters using low-cost sources featuring tenability, reconfigurability and negative coefficients," Opt. Exp., vol. 13, pp. 1412-1417, Feb 2005. 